\title{
Is BCG associated with reduced incidence of COVID-19? A meta-regression of global data from 160 countries
}

\author{
Melvin Joy $^{\text {a }}$, B. Malavika ${ }^{\text {a }}$ Edwin Sam Asirvatham ${ }^{\mathrm{b}}$, Thambu David Sudarsanam ${ }^{\mathrm{c}}$, \\ L. Jeyaseelan ${ }^{a, *}$ \\ ${ }^{a}$ Department of Biostatistics, Christian Medical College, Vellore, Tamil Nadu, 632 002, India \\ ${ }^{\mathrm{b}}$ Health Systems Research India Initiative (HSRII), Thiruvananthapuram, Kerala, India \\ ${ }^{\mathrm{c}}$ Department of Medicine, and Clinical Epidemiology Unit, Christian Medical College, Vellore, Tamil Nadu, 632 004, India
}

\section{A R T I C L E I N F O}

\section{Keywords:}

BCG Vaccine

COVID-19

Global burden of COVID-19

Meta-regression

Pandemic

\begin{abstract}
A B S T R A C T
Background: Global research is running towards to find a vaccine to stop the threat of the COVID-19. The Bacillus Calmette-Guérin (BCG) vaccine that prevents severe forms of tuberculosis is getting more attention in this scenario. The objective of our study was to determine the association between BCG vaccine coverage and incidence of COVID-19 at a national-level across the Globe.

Methods: The data of 160 countries were included in the study. Meta-regression was done to estimate the difference in the incidence of COVID-19 cases between countries with BCG vaccination coverage. BCG coverage was categorized as $\leq 70 \%,>70 \%$ and no vaccination. The analyses were carried out by adjusting for factors such as population density, income group, latitude, and percentage of the total population under age groups 15-64 and above 65 years of each country.

Results: The countries that had $\leq 70 \%$ coverage of BCG vaccine reported 6.5 (95\% CI: -8.4 to -4.5 ) less COVID19 infections per 10,000 population as compared to countries that reported no coverage. Those that had $>70 \%$ coverage reported 10.1 (95\% CI: -11.4 to -8.7 ) less infections per 10,000 population compared to those with no BCG countries.

Conclusion: Our analysis suggests that BCG is associated with reduced COVID-19 infections if the BCG vaccine coverage is over $70 \%$. The region-wise analyses also suggested similar findings, except the Middle East and North African region.
\end{abstract}

\section{Introduction}

There is evidence that the BCG (Bacillus Calmette Guerin) vaccine has been associated with beneficial, non-specific effects on the immune system. ${ }^{1}$ Studies suggest that the BCG vaccine induces the innate immune response which might reduce viraemia after exposure, leading to less severe COVID-19 and more rapid recovery. ${ }^{2}$ A randomized control trial suggests that BCG vaccine reduces mortality, attributable to protection against respiratory infections, as well as neonatal sepsis. ${ }^{3}$ Data on BCG vaccination status when analysed at a national-level along with incident cases of COVID-19, can give us an ecological idea of a possible protective effect, if any. The objective of our study was to determine the association between BCG vaccine coverage and incidence of COVID-19 at a national-level across the Globe.

\section{Methods}

Country level data on COVID-19 confirmed cases were obtained from European Center for Disease Control \& Prevention (ECDC). ${ }^{4}$ Data on BCG vaccination coverage was obtained from the WHO Global Health Observatory. ${ }^{5}$ The data related to other covariates were obtained from United Nations, Department of Economic and Social Affairs database. ${ }^{6}$ We included 160 countries that reported official data on BCG vaccination coverage (\%) and COVID-19 confirmed cases until May 31, 2020.

Meta-regression was done to estimate the difference in the incidence of COVID-19 cases between countries with BCG vaccination coverage. BCG coverage was categorized as $\leq 70 \%,>70 \%$ and no vaccination. We adjusted for population density, income group, latitude, and percentage of the total population under age groups 15-64 and above 65 years. The regression coefficients were presented in terms of number of cases per

\footnotetext{
* Corresponding author. Department of Biostatistics, Christian Medical College, Vellore, Tamil Nadu, 632 002, India.

E-mail addresses: melvinmj94@gmail.com (M. Joy), malavikababu@gmail.com (B. Malavika), aedwinsam@yahoo.com (E.S. Asirvatham), thambu@cmcvellore. ac.in (T.D. Sudarsanam), ljey@hotmail.com, prof.ljey@gmail.com (L. Jeyaseelan).
} 
Table 1

Results of meta-regression with incidence of COVID-19 as dependent variable by region \& income group.

$\begin{array}{lll}\begin{array}{l}\text { Number of Countries } \\ (\%)\end{array} & \begin{array}{l}\text { Regression Coefficient (95\% CI) } \\ \text { for BCG }\end{array} & \text { P value } \\ & \text { (Per 10,000 Population) }\end{array}$

(Per 10,000 Population)

\begin{tabular}{|c|c|c|c|}
\hline \multicolumn{4}{|c|}{ Global (160 Countries) } \\
\hline No BCG & $20(12.5)$ & Ref & \\
\hline $\begin{aligned} \leq & 70 \% \\
& \text { Covera }\end{aligned}$ & $9(5.6)$ & $-6.5(-8.4$ to -4.5$)$ & $<0.001$ \\
\hline $\begin{array}{l}>70 \% \\
\quad \text { Covera }\end{array}$ & $131(81.9)$ & $-10.1(-11.4$ to -8.7$)$ & $<0.001$ \\
\hline \multicolumn{4}{|c|}{ Region Wise } \\
\hline No BCG & $2(11.1)$ & Ref & \\
\hline $\begin{array}{l}\leq 70 \% \\
\quad \text { Covera }\end{array}$ & $1(5.6)$ & $-4.0(-6.7$ to -1.2$)$ & 0.004 \\
\hline $\begin{array}{l}>70 \% \\
\quad \text { Covera }\end{array}$ & $15(83.3)$ & $-4.2(-6.7$ to -1.6$)$ & 0.001 \\
\hline \multicolumn{4}{|c|}{ Europe \& Central Asia (40) } \\
\hline No BCG & $13(32.5)$ & & \\
\hline $\begin{aligned} \leq & 70 \% \\
& \text { Covera }\end{aligned}$ & $1(2.5)$ & $11.0(-12.5$ to 34.4$)$ & 0.359 \\
\hline $\begin{array}{l}>70 \% \\
\quad \text { Covera }\end{array}$ & $26(65.0)$ & $-18.4(-28.8$ to -8.0$)$ & 0.001 \\
\hline \multicolumn{4}{|c|}{ Latin America \& the Caribbean (27) } \\
\hline No BCG & $1(3.7)$ & Ref & \\
\hline $\begin{array}{l}>70 \% \\
\quad \text { Covera }\end{array}$ & $26(96.3)$ & $-14.3(-28.8$ to 0.2$)$ & 0.054 \\
\hline \multicolumn{4}{|c|}{ Middle East \& North Africa (18) } \\
\hline No BCG & $2(11.1)$ & Ref & \\
\hline $\begin{aligned} \leq & 70 \% \\
& \text { Covera }\end{aligned}$ & $1(5.6)$ & 254.4 (198.8 to 310.0$)$ & $<0.001$ \\
\hline $\begin{array}{l}>70 \% \\
\quad \text { Covera }\end{array}$ & $15(83.3)$ & $290.0(231.9$ to 348.1$)$ & $<0.001$ \\
\hline \multicolumn{4}{|c|}{ Income Group (World Bank) } \\
\hline No BCG & $2(4.2)$ & Ref & \\
\hline $\begin{array}{l}\leq 70 \% \\
\quad \text { Covera }\end{array}$ & $2(4.2)$ & $0.5(-9.8$ to 10.7$)$ & 0.929 \\
\hline $\begin{array}{l}>70 \% \\
\quad \text { Covera }\end{array}$ & $44(91.7)$ & $-3.2(-10.5$ to 4.2$)$ & 0.398 \\
\hline \multicolumn{4}{|c|}{ High Income (42) } \\
\hline No BCG & $18(42.9)$ & Ref & \\
\hline $\begin{aligned} \leq & 70 \% \\
& \text { Covera }\end{aligned}$ & $1(2.4)$ & $16.7(-12.2$ to 45.7$)$ & 0.258 \\
\hline $\begin{array}{l}>70 \% \\
\quad \text { Covera }\end{array}$ & $23(54.8)$ & $-17.7(-27.2$ to -8.1$)$ & $<0.001$ \\
\hline
\end{tabular}

Note 1: Adjusted for population density, income group, latitude, and percentage of total population under age groups 15-64 and above 65 years.

Note 2: Estimates cannot be calculated for South Asia and Sub-Saharan African region ( 8 and 47 countries respectively) because all these countries have implemented BCG policy. North American countries are not implementing BCG policy.

10000 population with $95 \%$ confidence interval by inverse variance method with random effect model, using the DerSimonian-Laird estimator for $\tau^{2}$. Statistical analyses were performed using Stata software, version 16.0 (StataCorp LLC).

\section{Results}

Of the 160 countries for whom BCG vaccination coverage data is available, 20 (12.5\%) did not use BCG, 5.6\% had a coverage of $\leq 70 \%$ and $82 \%$ had $>70 \%$ coverage (Table 1 ). The countries that had $\leq 70 \%$ coverage of BCG vaccine reported 6.5 (95\% CI: -8.4 to -4.5 ) less COVID-19 infections per 10,000 population as compared to countries that reported no coverage ( $\mathrm{p}<0.001$ ). Those that had $>70 \%$ coverage reported 10.1 (95\% CI: -11.4 to -8.7 ) less infections per 10,000 population compared to those with no BCG countries ( $p<0.001$ ).

Irrespective of the level of coverage, the East Asia and Pacific region countries reported 4 less infections per 10,000 population, as compared to countries without BCG vaccination strategy. Europe and Central Asian countries that had $>70 \%$ coverage, had 18.4 (95\% CI: -28.8 to -8.0 ) less infections per 10,000 population compared to no coverage $(\mathrm{p}<0.001)$. The Latin America and Caribbean countries that had $>70 \%$ coverage reported 14.3 (95\% CI: -28.8 to 0.2 ) per 10,000 less infections as compared to countries with no coverage.

The Middle East and North African countries, however, showed that those with $>70 \%$ BCG coverage had a higher number of infections as compared to no coverage. High-income countries, with $>70 \%$ coverage, had 17.7 (95\% CI: -27.2 to -8.1 ) less number of infections per 10,000 population $(\mathrm{p}<0.001)$ as compared to no coverage.

\section{Discussion}

Randomized trials of BCG vaccine prophylaxis against COVID-19 would be ideal. The WHO clinical trials portal reveals 5 such ongoing trials (NCT04414267, NCT04417335, EUCTR2020-002448-21-GR, IRCT20200411047019N1 and NL8609). Till these trial results are available, our meta-regression of country level data will give policy makers some idea of potential benefit of BCG vaccination in preventing COVID-19.

Our analysis suggests that BCG is associated with reduced COVID-19 infections if the BCG vaccine coverage is over $70 \%$. The region-wise analyses also suggested similar findings, except the Middle East and North African region. A large cohort from this region found no protective effect. $^{7}$ The spread of COVID-19 in the Middle East region is particularly alarming as these countries have continuously been considered as a hotspot for infectious diseases. ${ }^{8}$ The countries in this region are having larger number of international travelers every year for tourism, business, and pilgrimage. This population by genetics has higher prevalence for diabetes and hypertension that are risk factors for COVID-19 disease. ${ }^{9}$

Concluding that BCG protects an individual against COVID-19 may be an ecological fallacy. But this study adds impetus for us to carefully study the data from the ongoing BCG trials for making any policy and programmatic decisions.

\section{Funding}

This research did not receive any specific grant from funding agencies in the public, commercial, or not-for-profit sectors.

\section{Ethical Approval}

Not required.

\section{Declaration of competing interest}

None of the authors have conflicts of interest to report.

\section{References}

1 Curtis N, Sparrow A, Ghebreyesus TA, Netea MG. Considering BCG vaccination to reduce the impact of COVID-19. Lancet. 2020 May 16:395(10236):1545-1546.

2 O'Neill LAJ, Netea MG. BCG-induced trained immunity: can it offer protection against COVID-19? Nat Rev Immunol. 2020 Jun;20(6):335-337.

3 Aaby P, Roth A, Ravn H, et al. Randomized trial of BCG vaccination at birth to lowbirth-weight children: beneficial nonspecific effects in the neonatal period? J Infect Dis. 2011 Jul 15;204(2):245-252.

4 European Centre for Disease Prevention and Control [Accessed: 2020 May 31]. Available from https://www.ecdc.europa.eu/en/publications-data/download-toda ys-data-geographic-distribution-covid-19-cases-worldwide; 2020.

5 World Health Organization. Global Health observatory [Internet]. [Accessed: 2020 May 31]. Available from https://www.who.int/data/gho/data/indicators/indi cator-details/GHO/bcg-immunization-coverage-among-1-year-olds-(-).

6 United Nations, Department of Economics and Social Affairs, Population Division. World population prospects 2019. Online Edition. Rev. 1 [Internet]. [Accessed: 2020 May 31]. Available from https://population.un.org/wpp/Download/Standard/Popu lation/; 2019.

7 Hamiel U, Kozer E, Youngster I. SARS-CoV-2 rates in BCG-vaccinated and unvaccinated young adults. J Am Med Assoc. 2020 Jun 9;323(22):2340.

8 Buliva E, Elhakim M, Tran Minh NN, et al. Emerging and reemerging diseases in the world Health organization (WHO) eastern mediterranean region-progress, challenges, and WHO initiatives. Front Public Health. 2017 Oct 19:5:276.

9 Sawaya T, Ballouz T, Zaraket H, Rizk N. Coronavirus disease (COVID-19) in the Middle East: a call for a unified response. Front Public Health. 2020 May 19;8:209. 